\title{
Early Detection of Mild Alzheimer's Disease in Filipino Elderly: Validation of the Montreal Cognitive Assessment-Philippines (MoCA-P)
}

Jacqueline C. Dominguez, Jennifer Rose Soriano, Cely D. Magpantay, Mary Grace S. Orquiza, Wynette Marie Solis, Macario F. Reandelar Jr., Ma. Lourdes C. Joson

Memory Center, Institute for Neurosciences, St. Luke's Medical Center, Quezon City, Philippines

Email: jcdominguez@stluke.com.ph

Received 22 October 2014; revised 24 November 2014; accepted 3 December 2014

Copyright (C) 2014 by authors and Scientific Research Publishing Inc.

This work is licensed under the Creative Commons Attribution International License (CC BY).

http://creativecommons.org/licenses/by/4.0/

(c) (i) Open Access

\section{Abstract}

Background: The Montreal Cognitive Assessment (MoCA) is a useful instrument employed by clinicians to detect cognitive impairment and diagnose probable Alzheimer's Disease (AD) while in its early stages. Methods: A cross-sectional study was conducted to determine the diagnostic validity of the Philippine version of the MoCA (MoCA-P) among 1385 community-dwelling Filipino elderly from Marikina City, Metro Manila. Results: 509 controls and 97 elderly with probable AD and a Clinical Dementia Rating (CDR) global score of 0.5 were included in the analysis. Analysis of variance showed that the AD group was older $(p<0.05)$ and had fewer years of education $(p<$ 0.05). The optimal cut-off score to differentiate controls from those with probable AD was $20 / 21$, with a sensitivity of 0.835 and a specificity of 0.723 , and area under the curve (AUC) of 0.89 ( $p<$ 0.001). The positive and negative likelihood ratios were 3.01 and 0.23 , respectively; and pre- and post-test odds were 0.0951 and 0.2224 , respectively. Logistic regression showed that the odds of scoring $<20$ on the MoCA-P increased with advancing age and with education at $\leq 7$ years $(\mathrm{p}<$ 0.05). Two points are added to the MoCA-P score for those with $\leq 7$ years of education. Conclusion: The MoCA-P is a valid instrument for the early detection of mild AD among the Filipino elderly.

\section{Keywords}

Alzheimer Disease, Filipino, MoCA-P, Validation 


\section{Introduction}

Dementia is a global problem, with the majority of affected individuals living in developing countries in Asia [1]. Diagnosing dementia in its mild stage is a particular challenge in the Philippines where cognitive decline is generally dismissed as part of normal ageing, the elderly are excused from demanding mental activities, and access to specialists for timely diagnosis is limited. Moreover, most cognitive tests are developed in the English language and grounded on western cultures, hence performance on these tests are known to be influenced by language and culture. Age and education factors are also considered, where appropriate cut-off scores are often used to adjust for difference in these demographic factors [2]-[4]. With a view to address these, the validated Philippine version of the Mini-Mental Status Examination (MMSE-P) was developed [5]. Though commonly used, the MMSE does not detect mild cognitive impairment, thus a need for a more sensitive screening tool for detecting mild dementia.

The Montreal Cognitive Assessment (MoCA) was designed to detect mild cognitive impairment [6] in those with normal MMSE scores, and therefore stands as a better screening instrument for early detection of mild dementia. That it contains items that measure executive functions makes it reportedly useful in the assessment of Vascular Cognitive Impairment (VCI) [7] and in evaluating the cognitive functioning of patients with Parkinson's Disease [8]. The MoCA has been adapted and validated in various languages and cultures [9]-[12]. This study aimed to determine the diagnostic validity of the Philippine version of the MoCA (MoCA-P) [13] among urban community-dwelling elderly Filipinos, and the effects of age and education on MoCA-P performance.

\section{Methods}

\subsection{Study Design}

This was a cross-sectional study conducted in Marikina City, a part of Metro Manila, Philippines. Marikina City maintains a registry of their senior citizens which has a listing of 17,690 elderly. The study protocol was approved by the St. Luke's Medical Center Institutional Ethics Review Committee. Permission to adapt and validate the MoCA was obtained from Prof. Ziad Nasreddine.

\subsection{Participants}

The registry was randomly and proportionately sampled to make sure that all of Marikina City's 16 local government units were represented. A sample size of 1460 was calculated based on the reported sensitivity and specificity of the MoCA and an estimated 5\% prevalence of Alzheimer's Disease (AD).

\subsection{Procedure}

Prospective participants were invited to the community research site together with a reliable collateral informant. Informed consent was obtained from all participants or their next of kin when the participant was assessed by the investigators to be incompetent to sign the consent. Each participant underwent standard medical and neurological examination by a physician. The diagnosis of probable AD was made based on the National Institute of Non-Communicative Disorders and Stroke-Alzheimer Disease and Related Disorders Association (NINCDSADRDA) [14] and the stage of dementia was rated using the Clinical Dementia Rating (CDR) [15]. Neuropsychological testingwas conducted by trained psychologists using the MoCA-P, Geriatric Depression Scale (GDS) [16], and the Neuropsychiatric Inventory (NPI) [17]. The physicians and psychologists were blind to each other's ratings.

\subsection{Statistical Analyses}

Descriptive statistics and between-group comparisons using Analysis of Variance (ANOVA) were conducted to compare pertinent demographic and clinical characteristics of the study's participants. Sensitivity and specificity for each cut-off score in the MoCA-P score range was calculated. The optimal cut-off score for predicting probable AD was determined. A receiver operating characteristic (ROC) curve analysis with area under the curve (AUC) was used to determine the predictive value of the MoCA-P. Because of the lack of published prevalence indices of probable $\mathrm{AD}$ in the Philippines and the absence of an appropriate reference group, pre-test probability and pre-test odds were calculated using the prevalence of disease from the base sample size. Post-test odds were 
calculated using the likelihood ratio of the MoCA-P. Logistic regression analysis was used to determine the effect of education and age on MoCA-P performance. A p $<0.05$ was considered statistically significant in all analyses in this study. All statistical analyses were performed using Statistical Package for the Social Sciences (SPSS), version 17.0.

\section{Results}

Of the 1460 elderly who were recruited, 1,385 provided informed consent and participated in the study. Seventy five declined participation and 575 participants who were excluded from the study due to the following reasons: medical conditions that could affect test performance or confound AD diagnosis (e.g., significant hearing and/or visual impairment, history of stroke, depression, history of traumatic brain injury, hypothyroidism, other major psychiatric illness, Parkinson's Disease, seizures, use of anti-cholinergic drugs, and use of cognition-impairing medications); incomplete neuropsychological testing; primary language is not Filipino; difficulties in comprehending test instructions; and writing illiteracy that precluded performance on MoCA-P items that involved graphomotor output. In addition, 197 participants with Mild Cognitive Impairment (MCI) were excluded because they did not fulfill the criteria for probable AD.

The remaining 606 participants (509 controls, 97 participants with probable AD) comprised the final sample for analysis. They had a mean age of $68.9 \pm 6.51$ years, majority were female $(67.8 \%)$, and with mean years of education of $9.54 \pm 3.747$ years. The probable $\mathrm{AD}$ group was significantly older $(\mathrm{F}(2,799)=53.61, \mathrm{p}<0.05)$ and had lower education $(\mathrm{F}(2,795)=22.19, \mathrm{p}<0.05)$. The proportion of females who had probable AD was $15.8 \%$ and was not significantly different from that of males at $16.4 \%$ ( $\mathrm{p}$ value 0.852 ). The probable AD group was in the early stage of dementia as reflected in their mean CDR global score of $0.60 \pm 0.30$. The two groups did not differ with respect to the Modified Hachinski Ischemic Scale (MHIS) scores given that presence of stroke and vascular dementia were reasons for exclusion from the study $(\mathrm{F}(2,800)=0.83, \mathrm{p}=0.437)$. The mean MoCA-P score for the entire sample was $21.11 \pm 5.150$. The control group had significantly higher mean MoCA-P score and post-hoc analysis using Bonferroni adjustments showing no significant overlap of scores (Table 1). In both groups, MoCA-P scores decreased with increasing age. For every ten years increase in age, MoCA-P scores decreased by 1.5 and 2.4 points among controls and probable AD respectively. There was no significant difference in the relationship between age \& MoCA-P was seen between the two groups (Figure 1).

Sensitivity and specificity was calculated for every cut-off score (Table 2). A cut-off score of 19/20 had the

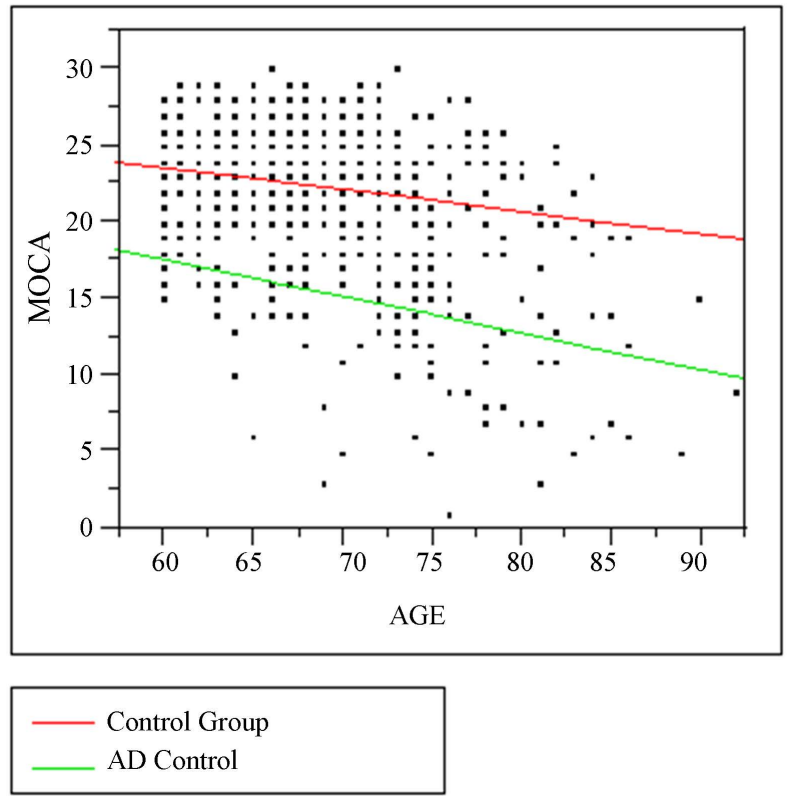

Figure 1. Scatterplot depicting relationship between age and MoCA-P scores among controls $\left(\mathrm{R}^{2}=0.05, \mathrm{~B}=-0.147\right)$ and probable $\mathrm{AD}$ population $\left(\mathrm{R}^{2}=0.06, \mathrm{~B}=-0.23\right)$. 
Table 1. Socio-demographic, CDR and MoCA-P profile according to clinical groups.

\begin{tabular}{ccccccc}
\hline \multirow{2}{*}{ Clinical Groups } & \multicolumn{5}{c}{ Socio-Demographic and Clinical Profile Characteristics } \\
\cline { 2 - 7 } & Age (Years) & Education (Years) & CDR $^{*}$ & CDR-SOB $^{*}$ & MHIS & MoCA-P $^{*}$ \\
Mean (sd)
\end{tabular}

Note: $\mathrm{CDR}=$ Clinical Dementia Rating; CDR-SOB $=$ CDR-Sum of Boxes; MHIS = Modified Hachinski Ischemic Scale; MoCA-P = Montreal Cogni-

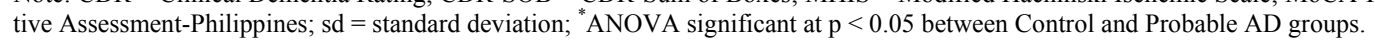

Table 2. Sensitivity and specificity of the MoCA-P at different cut-off scores.

\begin{tabular}{|c|c|c|}
\hline Cut-Off & Sensitivity & Specificity \\
\hline $10 / 11$ & 0.299 & 1 \\
\hline $11 / 12$ & 0.361 & 0.998 \\
\hline $12 / 13$ & 0.423 & 0.994 \\
\hline $13 / 14$ & 0.454 & 0.986 \\
\hline $14 / 15$ & 0.515 & 0.967 \\
\hline $15 / 16$ & 0.557 & 0.945 \\
\hline $16 / 17$ & 0.588 & 0.914 \\
\hline $17 / 18$ & 0.691 & 0.89 \\
\hline $18 / 19$ & 0.773 & 0.859 \\
\hline $19 / 20$ & 0.814 & 0.825 \\
\hline $20 / 21$ & 0.835 & 0.723 \\
\hline $21 / 22$ & 0.876 & 0.65 \\
\hline $22 / 23$ & 0.897 & 0.542 \\
\hline $23 / 24$ & 0.959 & 0.401 \\
\hline $24 / 25$ & 0.979 & 0.308 \\
\hline $25 / 26$ & 1 & 0.212 \\
\hline $26 / 27$ & 1 & 0.141 \\
\hline $27 / 28$ & 1 & 0.075 \\
\hline $28 / 29$ & 1 & 0.026 \\
\hline $29 / 30$ & 1 & 0.004 \\
\hline
\end{tabular}

Note: MoCA-P = Montreal Cognitive Assessment-Philippines.

highest sensitivity with good specificity at 0.814 and 0.825 , respectively. However, since the MoCA-P is being used as a screening test, the next score with better sensitivity $(0.835)$ was preferred to improve test sensitivity without much trade-off in specificity $(0.723)$, as seen in the ROC curve and AUC of 0.89 ( $\mathrm{p}<0.001)$ (Figure 2).

At this cut-off score, the positive likelihood ratio and negative likelihood ratio obtained for this cut-off score were 3.01 and 0.23 , respectively. With a pre-test probability of 0.0869 , pre-test odd was 0.0951 while post-test odd was 0.286 . Post-test probability was 0.2224 .

Results of the logistic regression analysis revealed that older age was significantly associated with a positive MoCA-P, defined as a score of $\leq 20(\mathrm{p}<0.05)$. Holding all variables constant, the odds of getting a positive MoCA-P score increased by $9 \%$ for every unit increase in age, or 2.5 times for every 10 -year increment in age (confidence interval $(\mathrm{CI})=1.065-1.130)$. 


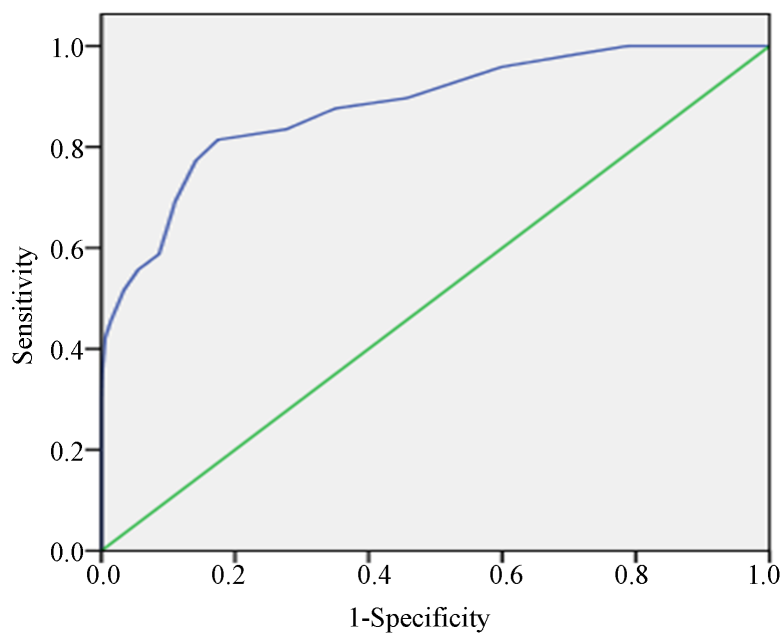

Figure 2. Receiver Operating Characteristic (ROC) Curve and Area Under the Curve (AUC) of the MoCA-P of 0.89 ( $\mathrm{p}<$ 0.001).

By logistic regression analysis, low education, defined as $\leq 7$ years of education, was also significantly associated with a positive MoCA-P score (i.e., fails the test) at $\mathrm{p}<0.05$. Holding all things constant, the odds of getting a positive MoCA-P score is 3.8 times more likely with low education $(\mathrm{CI}=2.649-5.563)$ (Table 3$)$. Additional logistic regression analysis revealed that the odds of probable $\mathrm{AD}$ increased by two times for every unit decrease in education, starting at 7 years (Table 4). Holding MoCA-P scores and age constant, lower education (i.e., $\leq 7$ years) increases the probability of being classified as having probable $\mathrm{AD}$ by almost 2 times $(\mathrm{CI}=1.059$ -2.852 ). Thus, 2 points is added to the total MoCA-P score and the cut-off year level of education was set at $\leq 7$ years. At this level, MoCA-P scores differed significantly when obtained from those with $\leq 7$ or $\geq 8$ years of education. Pre-test and post-test odds were calculated to be 15.7 and 47.16 , respectively. In a related analysis, education level specific cut-off was determined to be $17 / 18$ for $\leq 6$ years of education with a specificity, specificity and AUC of $0.70,0.89$ and 0.87 respectively and $19 / 20$ for $>6$ years of education with a sensitivity, specificity and AUC of $0.85,0.74$ and 0.85 respectively.

\section{Discussion}

The results of this validation study show that the MoCA-P can validly discriminate between early stage probable $\mathrm{AD}$ and control subjects at a cut-off score of 20/21. This cut-off is lower when compared to that of the original MoCA version which is $25 / 26$. Although most MoCA versions validated in various settings report the same cutoff scores as the original, different cut-off scores have been reported by others. A study done in a hospital setting on elderly urban-dwelling male veterans reported a cut-off score similar to our results, and they demonstrated that this cut-off score correlated significantly with other neuropsychological measures when compared to higher cut-off scores like 22/23 and 25/26 [18]. A lower cut-off of 22/23 was also recommended in a group of southwestern Caucasians [19] and this was reported to be optimal among co-Asians i.e. Koreans [20]. Both our sample and the Korean sample have lower mean years of education at $9.28 \pm 3.87$ and $8.3 \pm 3.7$, respectively. The Japanese sample of the MoCA-J [21] had $12.4 \pm 2.5$ years of education which is closer to the sample in the original version with $13.3 \pm 3.4$ years of education. Both have similar cut-off score. The new scoring rules of the original version now require adding 2 points to the total score for subjects with 4 - 9 years of education and 1 point for $10-12$ years of education. Our results recommend adding 2 points to the total MoCA-P score for those with $\leq 7$ years of education. Using the original cut-off and its education-based point adjustment is, therefore, not valid in our population as it may overestimate cognitive impairment and probable AD. Another way of handling the effect of education is to derive education-level specific cut-off scores. In our sample, a lower cut-off score for $\leq 6$ years of education was found. We recommend the use a single cut-off score for MoCA-P at 22/23 as it is easier to use in the community and in research. The negative influence of older age and lower education on neuropsychological test performance is confirmed in this study and education could be a reason for lower cut-off scores. 
Table 3. Effects of age and low education on MoCA-P scores.

\begin{tabular}{cccc}
\hline & Significance & OR & $95 \%$ CI \\
\hline Age & $\mathrm{p}<0.05$ & 1.097 & $1.065-1.130$ \\
Low education $(\leq 7$ years $)$ & $\mathrm{p}<0.05$ & 3.839 & $2.649-5.563$ \\
\hline
\end{tabular}

Note: MoCA-P = Montreal Cognitive Assessment-Philippines; $\mathrm{OR}=$ odds ratio; $\mathrm{CI}=$ confidence interval.

Table 4. Determination of additional points to MoCA-P scores for low education.

\begin{tabular}{lccc} 
& Significance & OR & $95 \%$ CI \\
\hline Low education $(\leq 7$ years $)$ & $\mathrm{p}<0.05$ & 1.738 & $1.059-2.852$ \\
\hline
\end{tabular}

Note: MoCA-P = Montreal Cognitive Assessment-Philippines; $\mathrm{OR}=$ odds ratio $; \mathrm{CI}=$ confidence interval.

In clinical settings, memory complaints are very common in the elderly, even those without probable AD. Such complaints may put physicians at a diagnostic dilemma and therefore, the MoCA-P can be very valuable in their evaluation. The pre-test probability of a person with memory complaints for having probable AD is 0.0869 or $8.69 \%$; but when MoCA-P is administered the probability increases to $0.2224 \%$ or $22.24 \%$ if the person tests positive (i.e., fails the test). A positive test, therefore, raises physicians' confidence to detect probable AD, thereby prompting early diagnosis. On the other hand, if the person tests negative (i.e., passes the test) such probability is markedly reduced to $0.0214 \%$ or $2.14 \%$. This negative test, likewise, increases physicians' confidence to assert absence of disease which is comforting to patients, though merits follow-up.

The ideal cognitive screening instrument in a primary care setting is brief and simple to administer, acceptable to patients, independent of education, culture, and language factors that confound performance, and broad in its coverage of cognitive domains of interest. Although the MoCA-P at the 20/21 cut-off is similar to the MMSE-P, which has a cut-off score of 23/24 in terms having same AUCs of 0.89 , the MoCA-P has the advantage of having more items that tap into attention, executive function, and visuo-spatial skills and is therefore more effective in detective mild impairment. However, the time needed to administer the MoCA-P was sometimes perceived to be lengthy given the number of tasks involved, which discouraged participation in some subjects. In light of this, it seems advisable to administer the MoCA-P when a patient fails the MMSE-P.

The cross-sectional design of this study involving a large sample size strengthens the generalizability of its findings. Our sample of 606 participants is fairly large for a validation study when compared to other MoCA validation studies where most sample sizes were less than 200 [22] [23]. Although probable AD comprised 16\% in this sample, it is not that small to make the ROC invalid. Moreover, that this study is drawn from a community-based sample makes it relatively more representative of the general population in terms of education, residential stability, and economic status. The MoCA-P cut-off score is, therefore, generally applicable to the Filipino elderly population.

\section{Conclusion}

The MoCA-P is a reliable and valid cognitive screening tool for the detection of mild probable AD among the Filipino elderly. The recommended cut-off score of $20 / 21$ has a sensitivity of 0.835 and a specificity of 0.723 . Its positive likelihood ratio and post test probability prove it to be useful in clinical practice.

\section{Acknowledgements}

The study team acknowledges the support of the Philippine Council for Health Research and Development (PCHRD) of the Philippine Department of Science and Technology, St. Luke's Medical Center Research and Biotechnology Division, Dr. Ziad Nasreddine, author of the MoCA who granted permission to adapt and validate the instrument, the staff of the Marikina Office of Senior Citizen's Affairs, the participants with their collateral informants, and Rachelle L. Holandez for her assistance in preparing the manuscript.

\section{References}

[1] Ferri, C.P., Prince, M., Brayne, C., Brodaty, H., Fratiglioni, L., Ganguli, M., et al. (2005) Global Prevalence of De- 
mentia: A Delphi Consensus Study. The Lancet, 366, 2112-2117. http://dx.doi.org/10.1016/S0140-6736(05)67889-0

[2] Crum, R.M., Anthony, J.C., Basset, S.S. and Folstein, M.F. (1993) Population-Based Norms for the Mini-Mental State Examination by Age and Educational Level. The Journal of the American Medical Association, 269, $2386-2391$. http://dx.doi.org/10.1001/jama.1993.03500180078038

[3] Zheng, L., Teng, E.L., Varma, R., Mack, W.J., Mungas, D., Lu, P.H. and Chui, H.C. (2012) Chinese-Language Montreal Cognitive Assessment for Cantonese or Mandarin Speakers: Age, Education, and Gender Effects. International Journal of Alzheimer's Disease, 2012.

[4] Das, S.K., Banerjee, T.K., Mukherjee, C.S., Bose, P., Biswas, A., Hazra, A., et al. (2006) An Urban Community-Based Study of Cognitive Function among Non-Demented Elderly Population in India. Neurology Asia, 11, 37-48.

[5] Ligsay, A.D. (2004) Validation of the Mini-Mental State Examination among the Filipino Elderly. M.Sc. Thesis, University of the Philippines, Manila.

[6] Nasreddine, Z.S., Phillips, N.A., Bédirian, V., Charbonneasu, S., Whitehead, V., Collin, I., et al. (2005) The Montreal Cognitive Assessment, MoCA: A Brief Screening Tool for Mild Cognitive Impairment. Journal of the American Geriatrics Society, 53, 695-699. http://dx.doi.org/10.1111/j.1532-5415.2005.53221.x

[7] Hachinski, V., Iadecola, C., Petersen, R.C., Breteler, M.M., Nyenhuis, D.L., Black, S.E., et al. (2006) National Institute of Neurological Disorders and Stroke-Canadian Stroke Network Vascular Cognitive Impairment Harmonization Standards. Stroke, 37, 2220-2241. http://dx.doi.org/10.1161/01.STR.0000237236.88823.47

[8] Kim, J.S., Kim, J.M., Kim, H.J., Yun, J.Y. and Jeon, B.S. (2013) Use of the Pill Questionnaire to Detect Cognitive Deficits and Assess Their Impact on Daily Life in Patients with Parkinson's Disease. Neurology Asia, 18, 369-375.

[9] Lee, J.Y., Dong, W.L., Cho, S.J., Na, D.L., Jeon, H.J., Kim, S.K., et al. (2008) Brief Screening for Mild Cognitive Impairment in Elderly Outpatient Clinic: Validation of the Korean Version of the Montreal Cognitive Assessment. Journal of Geriatric Psychiatry and Neurology, 21, 104-110. http://dx.doi.org/10.1177/0891988708316855

[10] Wen, H.B., Zhang, Z.X., Niu, F.S. and Li, L. (2008) The Application of Montreal Cognitive Assessment in Urban Chinese Residents of Beijing. Zonghua Nei Ke Za Zhi (Chinese Journal of Internal Medicine), 47, 36-39.

[11] Rahman, T.T. and El Gaafary, M.M. (2009) Montreal Cognitive Assessment Arabic Version: Reliability and Validity Prevalence of Mild Cognitive Impairment among Elderly Attending Geriatric Clubs in Cairo. Geriatrics \& Gerontology International, 9, 54-61.http://dx.doi.org/10.1111/j.1447-0594.2008.00509.x

[12] Montreal Cognitive Assessment Hong Kong Version (HK MoCA). http://www.mocatest.org/pdf_files/test/MoCA-Test-HongKong_2010.pdf

[13] Dominguez, J.C., Orquiza, M.S., Soriano, J.R., Magpantay, C.D., Esteban, R.C., Corrales, M.L. and Ampil, E.R. (2013) Adaptation of Montreal Cognitive Assessment for Elderly Filipino Patients. East Asian Archives of Psychiatry, 23, 8085.

[14] McKhann, G., Drachman, D., Folstein, M.F., Katzman, R., Price, D. and Stadlan, E.M. (1984) Clinical Diagnosis of Alzheimer Disease: Report of the NINCDS-ADRDA Work Group under the Auspices of the Department of Health and Human Services Task Force on Alzheimer Disease. Neurology, 34, 939-944. http://dx.doi.org/10.1212/WNL.34.7.939

[15] Morris, J.C. (1993) The Clinical Dementia Rating (CDR): Current Version and Scoring Rules. Neurology, 43, $2412-2414$. http://dx.doi.org/10.1212/WNL.43.11.2412-a

[16] Yesavage, J., Brink, T.L., Rose, T.L., Lum, O., Huang, V., Adey, M. and Leirer, V.O. (1983) Development and Validation of a Geriatric Depression Screening Scale: A Preliminary Report. Journal of Psychiatric Research, 17, 37-49. http://dx.doi.org/10.1016/0022-3956(82)90033-4

[17] Cummings, J.L., Mega, M., Gray, K., Rosenberg-Thompson, S., Carusi, D.A. and Gornbein, J. (1994) The Neuropsychiatric Inventory: Comprehensive Assessment of Psychopathology in Dementia. Neurology, 44, 2308-2314. http://dx.doi.org/10.1212/WNL.44.12.2308

[18] Waldron-Perrine, B. and Axelrod, B.N. (2012) Determining an Appropriate Cutting Score for Indication of Impairment on the Montreal Cognitive Assessment. International Journal of Geriatric Psychiatry, 27, 1189-1194. http://dx.doi.org/10.1002/gps.3768

[19] Luis, C.A., Keegan, A.P. and Mullan, M. (2009) Cross Validation of the Montreal Cognitive Assessment in Community-Dwelling Older Adults Residing in the Southeastern US. International Journal of Geriatric Psychiatry, 24, 197201. http://dx.doi.org/10.1002/gps.2101

[20] Lee, J.Y., Dong, W.L., Cho, S.J., Na, D.L., Jeon, H.J., Kim, S.K., et al. (2008) Brief Screening for Mild Cognitive Impairment in Elderly Outpatient Clinic: Validation of the Korean Version of the Montreal Cognitive Assessment. Journal of Geriatric Psychiatry and Neurology, 21, 104-110. http://dx.doi.org/10.1177/0891988708316855

[21] Fujiwara, Y., Suzuki, H., Yasunaga, M., Sugiyama, M., Ijuin, M., Sakuma, N., et al. (2010) Brief Screening Tool for Mild Cognitive Impairment in Older Japanese: Validation of the Japanese Version of the Montreal Cognitive Assessment. Geriatrics \& Gerontology International, 10, 225-232. http://dx.doi.org/10.1111/j.1447-0594.2010.00585.x 
[22] Memória, C.M., Yassuda, M.S., Nakano, E.Y. and Forlenza, O.V. (2013) Brief Screening for Mild Cognitive Impairment: Validation of the Brazilian Version of the Montreal Cognitive Assessment. International Journal of Geriatric Psychiatry, 28, 34-40. http://dx.doi.org/10.1002/gps.3787

[23] Gomez, F., Zunzunegui, M., Lord, C., Alvarado, B. and Garcia, A. (2013) Applicability of the MoCA-S Test in Populations with Little Education in Colombia. International Journal of Geriatric Psychiatry, 28, 813-820. http://dx.doi.org/10.1002/gps.3885 
Scientific Research Publishing (SCIRP) is one of the largest Open Access journal publishers. It is currently publishing more than 200 open access, online, peer-reviewed journals covering a wide range of academic disciplines. SCIRP serves the worldwide academic communities and contributes to the progress and application of science with its publication.

Other selected journals from SCIRP are listed as below. Submit your manuscript to us via either submit@scirp.org or Online Submission Portal.
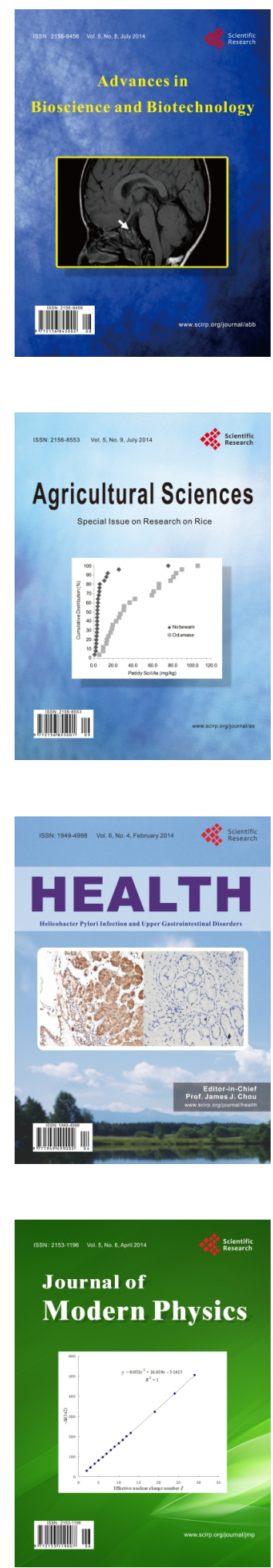
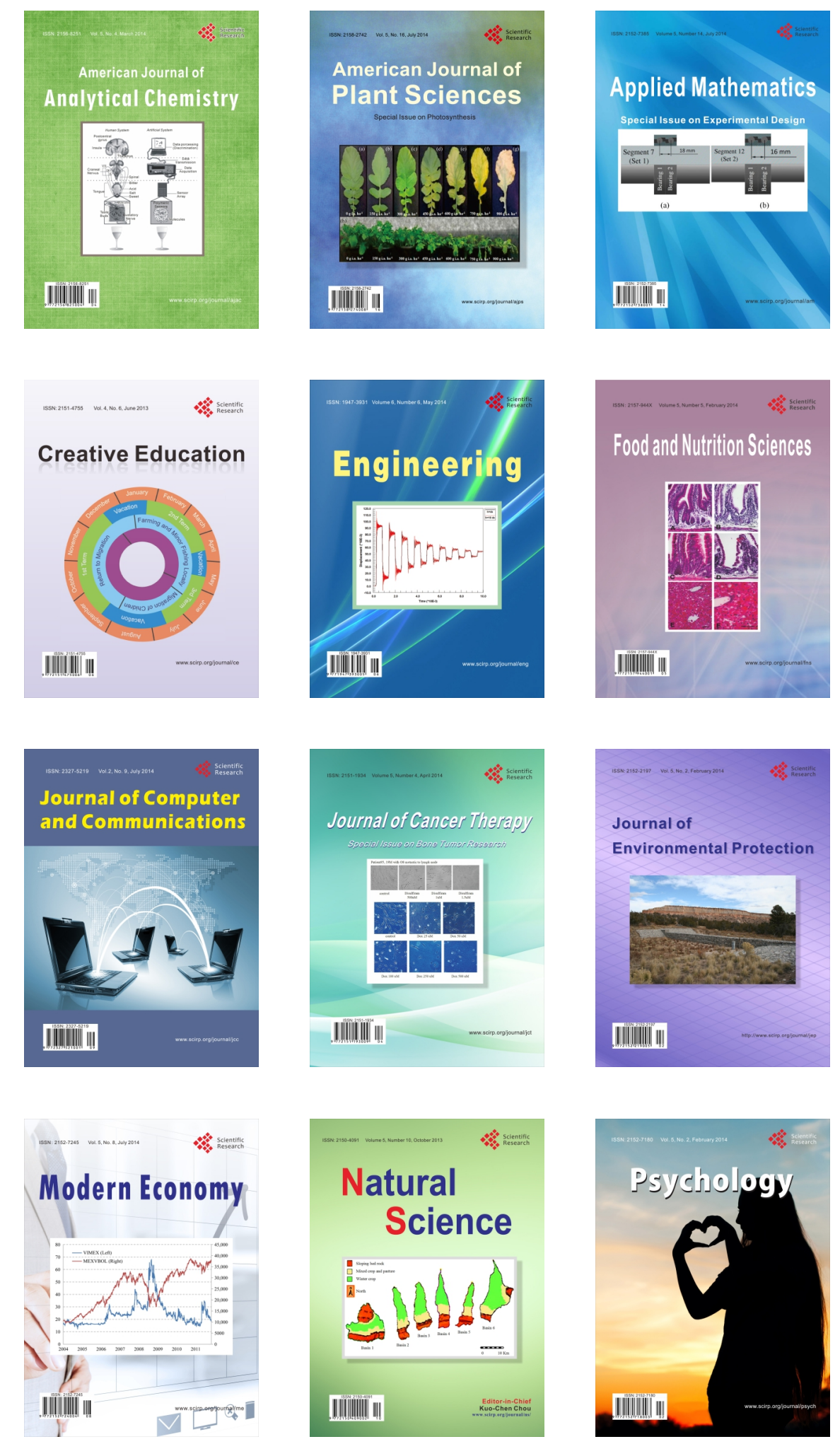